Kong. Res. J. 2(1) : 7-10, 2015

ISSN 2349-2694

Kongunadu Arts and Science College, Coimbatore.

\title{
PREPARATION AND CHARACTERIZATION POLY (VINYLIDENE FLUORIDE-TRIFLUOROETHYLENE) COPOLYMER THIN FILMS FOR ORGANIC FERROELECTRIC FIELD EFFECT THIN FILM TRANSISTORS.
}

\author{
Chandar Shekar, $\mathbf{B}^{1^{*}}$,, Sulana Sundari ${ }^{2}$, S. Sunnitha ${ }^{2}$ and C. Sharmila ${ }^{3}$ \\ ${ }^{1}$ Department of Physics, Kongunadu Arts and Science College, Coimbatore, Tamil Nadu, India. \\ ${ }^{2}$ The NEAR Foundation, The Nilgiris, Tamil Nadu, India. \\ ${ }^{3}$ Department of Physics, PSGR Krishnammal College for women, Coimbatore, Tamil Nadu, India. \\ *E-mail: chandar.bellan@gmail.com
}

\begin{abstract}
Polyvinylidene fluoride (PVDF) and Trifluoroethylene ((TrFE) are potential polymers which are used in acoustic transducers and electromechanical actuators because of their inherent piezoelectric response, as heat sensors because of their inherent pyroelectric response and as dielectric layer in organic thin film transistors. In the present study thin films of copolymer Poly(vinylidene fluoride-trifluoroethylene) were prepared by spin coating method for two different concentrations $2 \%$ to $8 \%$ and for various spin speeds from 2000 RPM to 5000 RPM. A P-type Si wafer was used as a substrate to deposit P(VDF-TrFE) thin films. 2butanone was used as a solvent to prepare $\mathrm{P}(\mathrm{VDF}-\mathrm{TrFE})$ solution. To study the annealing effect, the films were annealed for three different temperatures $50^{\circ} \mathrm{C}, 100^{\circ} \mathrm{C}$ and $175^{\circ} \mathrm{C}$. Ellipsometry was used to measure the thickness of the films. The identification of the films prepared was done by using FTIR spectrophotometer. The structure of the films was studied by using small angle XRD. The morphology of the coated surface was investigated using SEM. It is observed that the thickness of the film coated depends on concentration, spin speed and annealing temperature. The XRD spectrum indicated the amorphous nature with crystallites of very low dimension. SEM micrographs also conforms the predominantly amorphous nature of the film surface. The observed smooth surface with amorphous structure indicated that these films could be used as dielectric layer in organic ferroelectric field effect thin film transistors.
\end{abstract}

Keywords: PVDF -TrFE, Morphology, XRD,FTIR, SEM

\section{INTRODUCTION}

Ferroelectric field effect devices offer the possibility of non-volatile active memory elements. In the metal-ferroelectric-semiconductor field effect transistor, it is important for a ferroelectric material to have a suitable dielectric constant to enable the application of sufficient electric field to a ferroelectric film. Polyvinylidene fluoride (PVDF) and Trifluoroethylene ((TrFE) are potential polymers which are used in acoustic transducers and electromechanical actuators because of their inherent piezoelectric response, as heat sensors because of their inherent pyroelectric response, as an hole injecting interlayer in organic light emitting diodes (Jeon et al., 2010; Mahdi et al., 2014) as an interlayer in organic solar cells to improve the efficiency through fluorinated interlayer induced crystallization (Jeon et al., 2009; Xiao et al., 2013) and as dielectric layer in organic ferroelectric thin film transistors (Chang et al., 2009; Jung et al., 2010; Yoon et al., 2010). As a dielectric layer in organic ferroelectric field effect transistor structure along with suitable dielectric constant value it need to satisfy various constraints concerning band offsets, limits on charge traps, processability, reproducibility, stability against degradation, small leakage current, high breakdown potential, smooth surface and amorphous nature. In the present study effect of annealing on the structure and morphology of Polyvinylidene fluoride-trifluoroethylene (50/50) copolymer thin films prepared by spin coating was carried out with a view to use P(VDF-TrFE) film as an efficient dielectric layer in organic ferroelectric thin film transistors.

\section{MATERIALS AND METHODS}

Conventional P(VDF-TrFE) (50/50) copolymer was dissolved in 2-2-butanone to form the solution of concentrations $2 \%$ and $8 \%$. The solution was spun on Boron doped Si (100) substrate (p-type Si) for $20 \mathrm{Sec}$. at room temperature to prepare P(VDFTrFE) thin films. Before depositing P(VDF-TrFE), the surface preparations of silicon wafers were done by degreasing with organic solvents such as trichloroethylene (TCE) and ethanol and then followed by a rinse in deionized (DI) water. After spin coating process, the samples were dried in the vacuum chamber to evaporate the solvent remained 
in the film. The films were annealed in Ar ambient. The P(VDF-TrFE) films coated were identified by using a FTIR spectrometer. Spectroscopic ellipsometry (SE, J.A. Woolam Co., Model WVASE32) was used to measure the thickness of the films coated on Si substrate. The surface morphologies of the as deposited and annealed P(VDF-TrFE) films were investigated by means of SEM (FEI company, XL-305).

\section{RESULTS AND DISCUSSION}

To determine the amount of coating, P(VDFTrFE) (50/50) solution of $2 \%$ to $8 \%$ concentrations was chosen. Fig.1 shows the variation of film thickness with spin speed ranging from 2000 RPM to 5000 RMP for various concentrations (wt.\%) $2,4,6,8$ and 10 . For the concentrations of $2 \%$ to $8 \%$, the thickness of the film decreases with the increase in spin speed. For $2 \%$ concentration the variation in thickness is less when the spin speed increases above 3000 RPM. As the concentration increases, the variation in thickness coated with spin speed increases and it is much pronounced at $10 \%$ concentration.

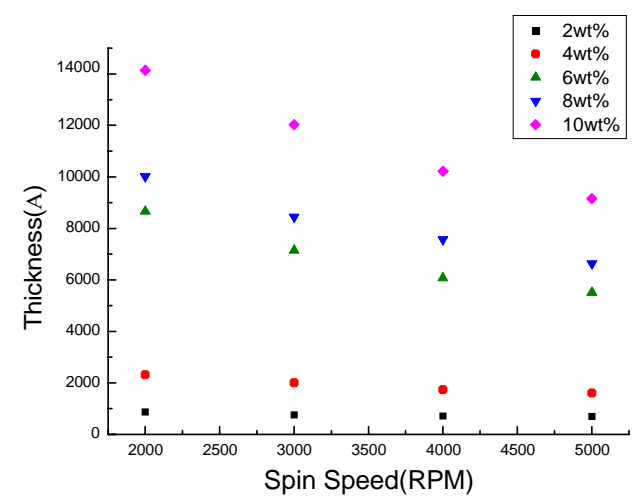

Fig. 1. Variation of thickness with spin speed for various concentrations.

Fig. 1 shows the thickness of the as grown and annealed films for various concentrations ranging from $2 \%$ to $8 \%$. The figure shows that the thickness of the as grown films increases from $752 \AA$ to $14000 \AA$ as the concentration increases from $2 \%$ to $8 \%$. For all the concentration range studied, the film thickness decreases with the increase of annealing temperature.

Fig.2 shows the FTIR spectrum PVDF-TrFE thin film of as grown $\left(8450 \AA\right.$ ) and annealed at $50^{\circ} \mathrm{C}$, $100^{\circ} \mathrm{C}$ and $175^{\circ} \mathrm{C}$.

The peak observed at $840 \mathrm{~cm}^{-1}$ is assigned to CF stretching where as the peaks observed at $890 \mathrm{~cm}^{-1}$ is assigned to $\mathrm{C}-\mathrm{H}$ bending.
The X-ray diffraction pattern of P(VDF-TrFE) film of thickness $8450 \AA$ is as shown in the Fig.3. The diffraction pattern indicates the predominantly amorphous nature with large diffraction maxima that decreases at large diffraction angles. The shape of the first main maximum indicates the ordered packing of the polymer chains. The intensity and shape of the second maxima are related to the effect of ordering inside the main chains. The prominent peaks observed around $19^{\circ}$ indicates the crystalline structure of spin coated PVDF-TrFE film (Nguyen et al., 2007). The observed broad humps in the XRD spectrum also indicate the presence of crystallites of very low dimensions.

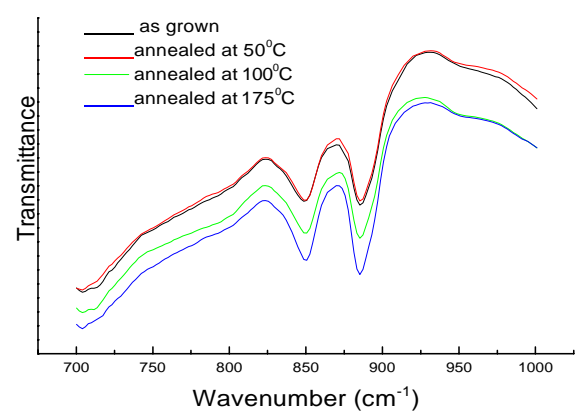

Fig. 2. FTIR spectrum of PVDF-TrFE thin film of thickness 8450 Å.

The crystal structure of P(VDF-TrFE) is normally related to the composition (mole ratio of $\mathrm{P}(\mathrm{VDF} / \mathrm{TrFE})$ of the copolymer and the annealing process. In the $\beta$ crystalline phase of P(VDF-TrFE), the unit cell is orthorhombic, with each chain aligned and packed with the CF2 groups parallel to the baxis. Figure 3 shows the XRD pattern of P(VDF-TrFE) for different annealing temperatures (not annealed

$\left(27{ }^{\circ} \mathrm{C}\right)$ to $\left.175^{\circ} \mathrm{C}\right)$ to obtain information on the degree of the crystalline structure of the copolymer thin films. Characteristic peaks, associated with the $\beta$ phase, appearing at $2 \theta=19^{\circ}$, are assigned to $(110 / 200)$ reflection planes. An elevated diffraction peak indicates a high percentage of the crystalline structure in the $\beta$ phase. One can note from the XRD result that the peak intensity at unannealed $\left(27{ }^{\circ} \mathrm{C}\right)$ was one of the lowest, because the annealing temperature was below the Curie temperature of the materials, which is not enough to align the chains. However, the highest intensity peak was achieved above $100{ }^{\circ} \mathrm{C}$, which means that the crystalline structure of the copolymer thin film is the highest. The Curie temperature of $\mathrm{P}(\mathrm{VDF}-\mathrm{TrFE})$ is around 90 ${ }^{\circ} \mathrm{C}$, where the $\beta$ phase starts to change above $100{ }^{\circ} \mathrm{C}$. The crystalline structure of the thin film obtained 
when annealed above $100{ }^{\circ} \mathrm{C}$ will be more favorable for the ferroelectric, pyroelectric and dielectric properties. This is due to the high crystalline structure exhibited by the composite thin film.

Surface morphology of dielectric layer is very important because it affects the property of the semiconductor layer coated over it. Fig. 4a-d shows the SEM image of the P(VDF-TrFE) films annealed at different temperatures. The film surface of as grown and films annealed at $50^{\circ} \mathrm{C}$ and $100^{\circ} \mathrm{C}$ is compact. Few pits are found on the surface. No pin holes, cracks and dendritic features are found in the surface. Macroscopic granular chains appear at the surface in the stretching direction of P(VDF-TrFE) film annealed at $175^{\circ} \mathrm{C}$. The granular structures (grooves) vary in size from approximately few nanometers to twenty nanometers.

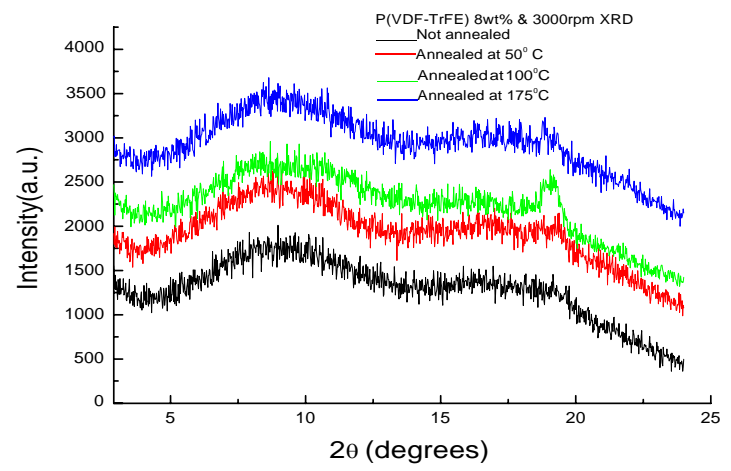

Fig. 3. XRD pattern of as grown and films annealed at $50^{\circ} \mathrm{C}, 100^{\circ} \mathrm{C} \& 175^{\circ} \mathrm{C}$ for 60 seconds.

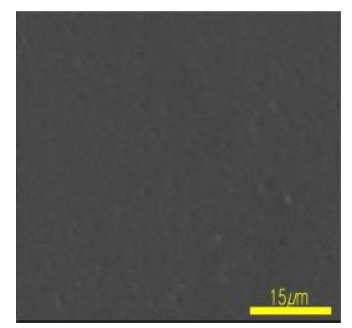

(a) As deposited

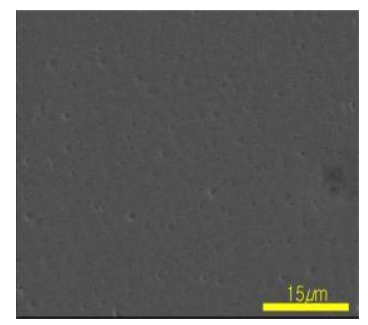

(c) Annealed at $100{ }^{\circ} \mathrm{C}$

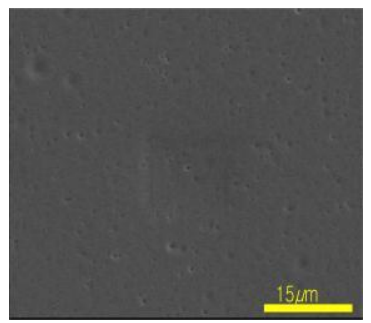

b) Annealed at $50^{\circ} \mathrm{C}$

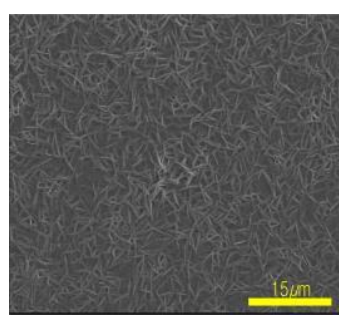

(d) Annealed at $175{ }^{\circ} \mathrm{C}$
Fig. 4. SEM image of a) as grown, b) $50{ }^{\circ} \mathrm{C}$ annealed, c) $100{ }^{\circ} \mathrm{C}$ annealed and d) $175^{\circ} \mathrm{C}$ annealed samples.
The surface morphology of both as grown (unannealed) and films annealed at $50^{\circ} \mathrm{C}$ and $100{ }^{\circ} \mathrm{C}$ is quite homogeneous and amorphous nature. Only films annealed at $175^{\circ} \mathrm{C}$ showed randomly ordered needle-like crystal domains of the PVDF-TrFE layer with a width of approximately $5 \mathrm{~nm}$ to $20 \mathrm{~nm}$. This indicates crystalline phase formation during thermal annealing process. As the annealing temperature increases, intrinsic changes in the microstructure of PVDF-TrFE as well as in interface are expected. These intrinsic changes may lead to the rougher and corrugated interfaces at the $\mathrm{p}-\mathrm{Si}$ substrate sides. Similar needle like structure for PVDF-TrFE film with a domain width of $40 \mathrm{~nm}$ to $200 \mathrm{~nm}$ was reported by Chang et al., 2009.

As deposited and films annealed up to $100{ }^{\circ} \mathrm{C}$ produced the morphology of the undefined crystalline structure. Significant elongated crystalline structures were clearly observed when the annealing temperature was increased to $175^{\circ} \mathrm{C}$. In general, $\mathrm{P}(\mathrm{VDF}-\mathrm{TrFE})$ thin films are annealed between the Curie and melting temperatures in order to induce the crystalline structure. In the paraelectric phase, the chain mobility is higher compared to that of the ferroelectric phase. It favors the lowest energy conformation (all trans), because as the temperature increases, the chain mobility increases as a function of temperature. As a result, the molecular chains prefer being oriented in parallel to the substrate, and rod-like crystals are observed when the annealing temperature reached $175^{\circ} \mathrm{C}$, as shown in Figure $4 \mathrm{~d}$. This suggests that small crystallites undergo a transition into a paraelectric phase. They grew by incorporating surrounding non-crystalline molecules and thus contributed to an increase in the crystalline structure. On the other hand, annealing above $100^{\circ} \mathrm{C}$ resulted in the formation of acicular grains (needlelike crystals) in edge-on lamellae, as shown in Figure $4 \mathrm{~d}$. Near the melting point, the morphology changes drastically, due to the small crystals being partially fused and recrystallized. In such a way, the chain axis is reoriented normal to the substrate surface. This implies that annealing above $100^{\circ} \mathrm{C}$ induced growth in the crystalline structure. The significant change in the morphology of the surface for the thin films annealed above $100^{\circ} \mathrm{C}$, can be clearly observed with the combination of some defects, such as a crack in the surface, as shown in Figure 4d. The defects will lead to a decrease in the electrical properties of the material.

\section{CONCLUSIONS}

P(VDF-TrFE) thin films of around $50 \mathrm{~nm}$ to $1200 \mathrm{~nm}$ have been prepared by spin coating with a 
spin speed between 2000 RPM and 5000 RPM. Surface morphology and structure of the PVDF-TrFE thin films have been studied by SEM and XRD. No pin holes were found in the surface. Both as grown and films annealed up to $100^{\circ} \mathrm{C}$ showed smooth surface. The observed topographical features of the as grown and annealed films indicated the predominantly amorphous nature of the films studied. XRD indicated the presence of crystallites of very low dimension in the predominantly dominated amorphous phase. The effect of annealing temperature on the crystalline structure of P(VDFTrFE) thin films has been investigated with a wide range of treated temperature $\left(27-175{ }^{\circ} \mathrm{C}\right)$ below and above the Curie temperature to optimize the crystalline structure. The annealing temperature is an essential parameter that can be used to enhance the crystalline structure of the thin films and the related electrical properties (pyro-, ferro- and dielectric). From the obtained results, we found that the optimized crystalline structure was achieved at around $175^{\circ} \mathrm{C}$ when the thin films were annealed in a vacuum chamber oven. The thin films show very sharp and high intensity XRD peaks with a dominant $\beta$ phase.

If the thin films were annealed with an oven, it required a higher optimum annealing temperature, which is about $175^{\circ} \mathrm{C}$. This annealing temperature is much closer to the melting point of the material and, thus, can cause many defects in the sample. The lamella length increases at $175{ }^{\circ} \mathrm{C}$ with cracks appearing on the surface of the thin film. The thin films also had poor ferroelectric and pyroelectric properties with high dielectric loss when annealed below the Curie temperature. Thus, the technique of annealing the thin film above $100{ }^{\circ} \mathrm{C}$ with a vacuum chamber oven can be used to achieve P(VDF-TrFE) thin film with improved ferroelectric, dielectric and pyroelectric properties, which is suitable for many applications, such as energy storage, sensors and actuators. The observed thermal stability, amorphous and smooth surface implies that thin films of P(VDF -TrFE) formed by spin coating can be used as sensors, solar cells and an efficient dielectric layer in ferroelectric organic thin film transistors.

\section{REFERENCES}

Chang, J., C.H. Shin, Y.J. Park, S.J. Kang, H.J. Jeong, K.J. Kim, C.J. Hawker, T.P. Russell, D. Y. Ryu and C. Park. (2009). Organic Electronics 10: 849-856.

Jeon, S.O., K.S. Yook and J.Y. Lee. (2009). Organic Electronics 10: 1583-1589.

Jeon, S.O., K.S. Yook and J.Y. Lee. (2010). Journal of Luminescence 130: 1708-1710.

Jung, S.W., J.K. Lee, Y.S. Kim, S.M. Yoon, I.K. You, B.G. Yu and Y.Y. Noh. (2010). Current Applied Physics 10: $58-61$.

Maude, R.I., W. C. Gan and W.H.A. Majid. (2014). Sensors 14: 19115-19127.

Nguyen, C.A., P.S. Lee and S.G. Mhaisalkar. (2007). Organic Electronics 8: 415-422.

Xiao, Z., Q. Dong, P. Sharma, Y. Yuan, B. Mao, W. Tian, A. Gruverman and J. Huang. (2013). Advanced Energy Materials 3: 1581- 1588.

Yoon, S.M., S.W. Jung, S.H. Yang, C.W. Byun, C.S. Hwang, S.H.K. Park and H. Ishiwara. (2010). Organic Electronics 11: 1746-1752. 